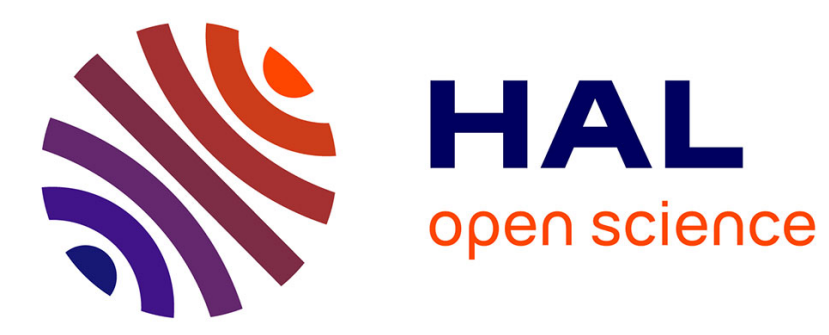

\title{
An inverse problem involving two coefficients in a nonlinear reaction-diffusion equation
}

\author{
Michel Cristofol, Lionel Roques
}

\section{To cite this version:}

Michel Cristofol, Lionel Roques. An inverse problem involving two coefficients in a nonlinear reactiondiffusion equation. 2010. hal-00592268

\author{
HAL Id: hal-00592268 \\ https://hal.science/hal-00592268 \\ Preprint submitted on 11 May 2011
}

HAL is a multi-disciplinary open access archive for the deposit and dissemination of scientific research documents, whether they are published or not. The documents may come from teaching and research institutions in France or abroad, or from public or private research centers.
L'archive ouverte pluridisciplinaire HAL, est destinée au dépôt et à la diffusion de documents scientifiques de niveau recherche, publiés ou non, émanant des établissements d'enseignement et de recherche français ou étrangers, des laboratoires publics ou privés. 


\title{
An inverse problem involving two coefficients in a nonlinear reaction-diffusion equation
}

\author{
Michel Cristofol ${ }^{\mathrm{a}, 1}$, Lionel Roques ${ }^{\mathrm{b}}$, \\ ${ }^{a}$ Laboratoire d'Analyse Topologie Probabilités, CNRS UMR 6632, Universités d'Aix-Marseille, Marseille, France \\ ${ }^{\mathrm{b}}$ UR 546 Biostatistique et Processus Spatiaux, INRA, F-84000 Avignon, France \\ Received *****; accepted after revision +++++ \\ Presented by $£ £ £ £ £$
}

\begin{abstract}
This Note deals with a uniqueness and stability result for a nonlinear reaction-diffusion equation with heterogeneous coefficients, which arises as a model of population dynamics in heterogeneous environments. We obtain a Lipschitz stability inequality which implies that two non-constant coefficients of the equation, which can be respectively interpreted as intrinsic growth rate and intraspecific competition coefficients, are uniquely determined by the knowledge of the solution on the whole domain at two times $t_{0}$ and $t_{1}$ and on a subdomain during a time interval which contains $t_{0}$ and $t_{1}$. This inequality can be used to reconstruct the coefficients of the equation using only partial measurements of its solution.

To cite this article: M. Cristofol and L. Roques, C. R. Acad. Sci. Paris, Ser. I*** (****).
\end{abstract}

\section{Résumé}

Reconstruction simultanée de deux coefficients dans une équation de réaction-diffusion non-linéaire. Dans cette Note, nous présentons un résultat d'unicité et de stabilité pour une équation de réaction-diffusion non linéaire et à coefficients hétérogènes, intervenant notamment dans des modèles de dynamique des populations. Nous établissons une inégalité du type Lipschitz impliquant que la connaissance de la solution de l'équation sur tout le domaine d'étude à des temps $t_{0}$ et $t_{1}$, ainsi que sa connaissance sur un sous-domaine durant un intervalle de temps contenant $t_{0}$ et $t_{1}$, détermine de façon unique deux coefficients hétérogènes de l'équation.

Pour citer cet article : M. Cristofol and L. Roques, C. R. Acad. Sci. Paris, Ser. I*** (****).

Email addresses: cristo@cmi.univ-mrs.fr (Michel Cristofol ), lionel.roques@inra.fr (Lionel Roques).

1 Corresponding author. 


\section{Version française abrégée}

L'équation de réaction-diffusion (1), dans laquelle $u(t, x)$ correspond à une densité de particules à un temps $t$ et une position $x$, fut introduite par Fisher et Kolmogorov et al. [6,9] pour modéliser un problème de génétique des populations. Depuis, ce modèle est utilisé dans des domaines allant de l'écologie à la cytologie. Nous nous intéressons ici à sa version hétérogène. Alors que le coefficient de diffusion est supposé constant, nous faisons en effet l'hypothèse que les coefficients de croissance intrinsèque, $\mu(x)$, et de compétition intraspécifique, $\gamma(x)$, peuvent dépendre de la variable d'espace. Ces deux coefficients jouent un rôle essentiel en dynamique des populations. Nous les supposons non connus, notre objectif étant de les retrouver à partir de mesures partielles de $u(t, x)$, via une inégalité de stabilité. A notre connaissance, dans la littérature existante, de tels résultats de stabilité portent uniquement sur un coefficient. En se plaçant dans un domaine borné $\Omega$, et en utilisant des résultats de régularité parabolique (voir [7]), le principe du maximum ainsi que le lemme de Hopf, nous démontrons tout d'abord trois lemmes préliminaires (1.1, 2.1 et 2.2) donnant des estimations a priori des solutions de (1), indépendantes des coefficients de l'équation. Nous établissons ensuite trois inégalités de Carleman (avec des poids spéciaux) associées aux systèmes (4), (5) et (6). Le principal résultat (12) en découle. Il implique que si deux coefficients $\tilde{\mu}$ et $\tilde{\gamma}$ sont tels que la solution $\tilde{u}$ de (1), où $\mu$ et $\gamma$ sont respectivement remplacés par $\tilde{\mu}$ et $\tilde{\gamma}$, est proche de $u$ sur $\Omega$ aux temps $t_{0}$ et $t_{1}$ et dans un sous-domaine $\omega$ durant un intervalle de temps $] t_{0}-\delta, t_{1}+\delta[$, alors $\tilde{\mu}$ est proche de $\mu$ et $\tilde{\gamma}$ est proche de $\gamma$.

\section{Introduction and main results}

The idea of modelling population dynamics with reaction-diffusion models has begun to develop at the beginning of the $20^{\text {th }}$ century, with random walk theories of organisms. Then independently, Fisher [6] and Kolmogorov, Petrovsky, Piskunov [9] used a reaction-diffusion equation as a model for population genetics. The corresponding equation is $u_{t}=D \nabla^{2} u+u(\mu-\gamma u)$, where $u=u(t, x)$ is the population density at time $t$ and space position $x, D$ is the diffusion coefficient, and $\mu$ and $\gamma$ respectively correspond to the constant intrinsic growth rate and intraspecific competition coefficients. In the 80 's, this model has been extended to heterogeneous environments by Skellam [16]:

$$
u_{t}=D \nabla^{2} u+u(\mu(x)-\gamma(x) u), \text { for } t>0,
$$

in a bounded and smooth domain $\Omega \subset \mathbb{R}^{N}$. Recently, this model revealed that the heterogeneous character of the environment played an essential role on species persistence and spreading, in the sense that for different spatial configurations of the environment, a population can survive or become extinct and spread at different speeds, depending on the habitat spatial structure ([4],[10],[11],,[13],[14],,[15],[17]). In a previous work [1] assuming that $\gamma$ was constant, we stated a stability inequality which enabled us to successfully recover $\mu(x)$, using the following measurements: (i) $\mu(x)$ is known and equal to a constant near the boundary $\partial \Omega$; (ii) the density $u(0, x)$ is known in $\Omega$ at $t=0$; (iii) the density $u(t, x)$ is known and equal to 0 for $(t, x) \in[0, T] \times \partial \Omega$ for some $T>0$; (iv) the density $u(t, x)$ is known for $(t, x) \in\left(t_{0}, t_{1}\right) \times \omega$, for some times $0<t_{0}<t_{1}<T$ and a subset $\omega \subset \subset \Omega$; (v) the density $u\left(\frac{t_{0}+t_{1}}{2}, x\right)$ is known for all $x \in \Omega$.

Here, our aim is to obtain a stability inequality which enables to simultaneously recover both coefficients $\mu(x)$ and $\gamma(x)$, and to prove their uniqueness, provided they belong to a particular subset of $C^{\infty}(\bar{\Omega})$, given the following information: (i') $\mu(x)$ and $\gamma(x)$ are known near the boundary $\partial \Omega$; (ii') the density $u_{i}(x)=u(0, x)$ is known in $\Omega$ at $t=0$; (iii') $u$ is known and satisfies Neumann boundary conditions in $[0, \infty) \times \partial \Omega$; (iv') the density $u(t, x)$ is known in a finite time interval and in a subset $\omega \subset \subset \Omega$; (v') the densities $u\left(t_{0}, x\right)$ and $u\left(t_{1}, x\right)$ are known at two fixed times $t_{0}, t_{1}$ and for all $x \in \Omega$. 
Few works are related to the reconstruction of several coefficients of reaction-diffusion equations. Furthermore, those works only deal with the reconstruction of source terms and initial conditions (see e.g. [3], [18]), or provide uniqueness results without stability [12].

The main tools used to establish these new results are Carleman estimate with special weights and parabolic estimates together with parabolic maximum principle and Hopf's lemma.

Let us make our hypotheses more precise. We define two subsets of $\mathbb{C}^{\infty}(\bar{\Omega})$ by $M_{1}:=\{\tilde{\mu}$ s.t. $\tilde{\mu}(x)=$ $\mu^{*}(x)$ if $d(x, \partial \Omega)<\varepsilon$, for all $\left.x \in \Omega\right\}$, and $\Gamma_{1}:=\left\{\tilde{\gamma}\right.$ s.t. $\tilde{\gamma}(x)=\gamma^{*}(x)$ if $d(x, \partial \Omega)<\varepsilon$, for all $\left.x \in \Omega\right\}$, for given functions $\mu^{*} \in C^{\infty}(\bar{\Omega})$ and $\gamma^{*} \in C^{\infty}(\bar{\Omega})$, and a small parameter $\varepsilon>0 ; d(x, \partial \Omega)$ corresponds to the distance from $x$ to $\partial \Omega$. Let $M_{2}$ and $\Gamma_{2}$ be two other subsets of $C^{\infty}(\bar{\Omega})$, defined by: $M_{2}:=\left\{\tilde{\mu}\right.$ s.t. $\mu^{-} \leq$ $\tilde{\mu} \leq \mu^{+}$on $\left.\bar{\Omega}\right\}$, for two given functions $\mu^{+}, \mu^{-}$in $M_{1}$ with $0<\mu^{-} \leq \mu^{+}$, and $\Gamma_{2}:=\left\{\tilde{\gamma}\right.$ s.t. $\gamma^{-} \leq \tilde{\gamma} \leq$ $\gamma^{+}$on $\bar{\Omega}$, for two given functions $\gamma^{-}$and $\gamma^{+}$in $\Gamma_{1}$ with $0<\gamma^{-} \leq \gamma^{+}$. Lastly, let $K \subset C^{\infty}(\bar{\Omega})$ be defined by $K:=\left\{\rho\right.$ s.t. $\left.\|\rho\|_{C^{5}(\bar{\Omega})} \leq m\right\}$, for some $m>0$.

We then define the state spaces $M$ and $\Gamma$ by $M:=M_{1} \cap M_{2} \cap K$, and $\Gamma:=\Gamma_{1} \cap \Gamma_{2} \cap K$. Note that, if $\varepsilon$ is chosen small enough and $m$ is chosen large enough, these sets are not empty.

Let us fix two couples $(\mu, \gamma)$ and $(\tilde{\mu}, \tilde{\gamma})$ in $M \times \Gamma$ and let $u, \tilde{u}$ be, respectively, the solutions of

$$
\left\{\begin{array} { l } 
{ \partial _ { t } u = D \Delta u + u ( \mu - \gamma u ) \text { in } ( 0 , \infty ) \times \Omega , } \\
{ \partial _ { \nu } u = 0 \text { on } [ 0 , \infty ) \times \partial \Omega , } \\
{ u ( 0 , \cdot ) = u _ { i } \text { in } \Omega , }
\end{array} \text { and } \left\{\begin{array}{l}
\partial_{t} \tilde{u}=D \Delta \tilde{u}+\tilde{u}(\tilde{\mu}-\tilde{\gamma} \tilde{u}) \text { in }(0, \infty) \times \Omega, \\
\tilde{u}=u \text { on }[0, \infty) \times \partial \Omega \\
\tilde{u}(0, \cdot)=u_{i} \text { in } \Omega .
\end{array}\right.\right.
$$

for some constant $D>0$ and some function $u_{i}$ in $C^{\infty}(\bar{\Omega})$ which verifies:

$$
u_{i}>0 \text { in } \Omega, \partial_{\nu} u_{i}=0 \text { on } \partial \Omega \text { and } 6 \sup _{\Omega} u_{i}<\inf _{\Omega} \mu^{-} / \sup _{\Omega} \gamma^{+} .
$$

The functions $u, \tilde{u}$ belong to $C_{1}^{2}([0, \infty) \times \bar{\Omega}) \cap C^{\infty}([\sigma, \infty) \times \bar{\Omega})$, for any $\sigma>0^{2}$.

Before stating our main theorem, let us state a preliminary lemma:

Lemma 1.1 It exists an interval $\mathcal{T}$ in $(0, \infty)$ such that, for any couple $\left(t_{0}, t_{1}\right)$ with $0<t_{0} \leq \inf \mathcal{T}<$ $\sup \mathcal{T} \leq t_{1}$, and for all $(\tilde{\mu}, \tilde{\gamma}) \in M \times \Gamma$,

$$
6 \sup _{x \in \bar{\Omega}} \tilde{u}\left(t_{0}, x\right)<\inf _{x \in \bar{\Omega}} \tilde{u}\left(t_{1}, x\right) .
$$

The interval $\mathcal{T}$ can be computed explicitly. The proof of this lemma uses hypothesis (3) on $u_{i}$.

Our main result is:

Theorem 1.2 For any $\omega \subset \subset \Omega$ and any time interval $\left(t_{0}, t_{1}\right)$ containing $\mathcal{T}$ it exists $\delta \in\left(0, t_{0}\right)$ and a constant $C$ such that for all $\mu, \tilde{\mu} \in M, \gamma, \tilde{\gamma} \in \Gamma$,

$$
\begin{gathered}
\|\mu-\widetilde{\mu}\|_{L^{2}(\Omega)}^{2}+\|\gamma-\widetilde{\gamma}\|_{L^{2}(\Omega)}^{2} \leq C G(u, \tilde{u}), \quad \text { with } \\
G(u, \tilde{u})=\|u-\widetilde{u}\|_{H^{2}\left(\left(t_{0}-\delta, t_{1}+\delta\right), L^{2}(\omega)\right)}^{2}+\left\|(u-\widetilde{u})\left(t_{0}, .\right)\right\|_{H^{2}(\Omega)}^{2}+\left\|(u-\widetilde{u})\left(t_{1}, .\right)\right\|_{H^{2}(\Omega)}^{2} .
\end{gathered}
$$

A straightforward corollary is a uniqueness result for the couple $(\tilde{\mu}, \tilde{\gamma})$, given $u\left(t_{0}, x\right), u\left(t_{1}, x\right)$ for $x \in \Omega$ and $u(t, x)$ for $t \in\left(t_{0}-\delta, t_{1}+\delta\right)$ and $x \in \omega$. Another practical consequence of Theorem 1.2 is to enable a numerical reconstruction of the unknown coefficients $\mu$ and $\gamma$, given the partial measurements (i)', (ii)', (iii)', (iv)', (v)' (see [1]).

In the sequel, we give a very schematic proof of this result. More details will be given in the forthcoming paper [2].

\footnotetext{
${ }_{2}$ The spaces $C_{j}^{i}([\sigma, \infty) \times \bar{\Omega})$ are spaces of functions on $[\sigma, \infty) \times \bar{\Omega}$ whose derivatives up to order $i$ in $x$ and order $j$ in $t$ are continuous. The regularity of $\tilde{u}$ follows from the hypothesis on $u_{i}$ and from the definition of $M_{1}$ and $\Gamma_{1}$ which lead to proper compatibility conditions.
} 


\section{Proof of Theorem 1.2 .}

We begin with a priori estimates on $\tilde{u}$, independent of the choice of the couple $(\tilde{\mu}, \tilde{\gamma}) \in M \times \Gamma$.

Lemma 2.1 For any $T>0$ it exists $r>0$ such that, for all $(\tilde{\mu}, \tilde{\gamma}) \in M \times \Gamma, \tilde{u} \geq r$ and $\partial_{t} \tilde{u} \geq$ $r$ on $[0, T] \times \bar{\Omega}$.

The next lemma states $C_{\alpha / 2}^{\alpha}$ boundary estimates on $\tilde{u}$ and its time derivatives.

Lemma 2.2 For any $T>0$ and $\sigma \in(0, T)$ it exists a constant $\widetilde{C}>0$, independent of the choice of $(\tilde{\mu}, \tilde{\gamma}) \in M \times \Gamma$, such that:

$$
\|\tilde{u}\|_{C_{1}^{2}([\sigma, T] \times \bar{\Omega})},\left\|\tilde{u}_{t}\right\|_{C_{1}^{2}([\sigma, T] \times \bar{\Omega})},\left\|\tilde{u}_{t t}\right\|_{C_{1}^{2}([\sigma, T] \times \bar{\Omega})} \leq \widetilde{C} .
$$

The main tools used to prove Lemmata 1.1 and 2.1 are comparison principles and Hopf's lemma.

Let $u$ (resp. $\widetilde{u})$ be the solution of $(2)$ associated to $(\mu, \gamma)(\operatorname{resp} .(\tilde{\mu}, \tilde{\gamma}))$. We set $U=u-\widetilde{u}$. The function $U$ satisfies:

$$
\left\{\begin{array}{l}
\partial_{t} U=D \Delta U+\mu U-\gamma U(\widetilde{u}+u)+\alpha \widetilde{u}-\beta \widetilde{u}^{2} \text { in }(0, \infty) \times \Omega, \\
U(t, x)=0 \text { on }[0, \infty) \times \partial \Omega \\
U(0, x)=0 \text { in } \Omega
\end{array}\right.
$$

where $\alpha=\mu-\tilde{\mu}$ and $\beta=\gamma-\tilde{\gamma}$. Using Lemma 2.1, we can set $y=\frac{U}{\widetilde{u}}$ and the previous system becomes

$$
\left\{\begin{array}{l}
\partial_{t} y=D \Delta y+\frac{2 D}{\widetilde{u}} \nabla \widetilde{u} \cdot \nabla y+y\left(\mu+D \frac{\Delta \widetilde{u}}{\widetilde{u}}-\frac{\partial_{t} \widetilde{u}}{\widetilde{u}}-\gamma(u+\tilde{u})\right)+\alpha-\beta \widetilde{u}, \text { in }(0, T] \times \Omega, \\
y(t, x)=0 \text { on }[0, T] \times \partial \Omega \\
y(0, x)=0 \text { on } \Omega .
\end{array}\right.
$$

We set $z=\partial_{t} y$. Writing $A=\mu+D \frac{\Delta \widetilde{u}}{\widetilde{u}}-\frac{\partial_{t} \widetilde{u}}{\widetilde{u}}-\gamma(u+\tilde{u})$, we get:

$$
\left\{\begin{array}{l}
\partial_{t} z=D \Delta z+\frac{2 D}{\widetilde{u}} \nabla \widetilde{u} \cdot \nabla z+A z+\partial_{t}\left(\frac{2 D}{\widetilde{u}} \nabla \widetilde{u}\right) \cdot \nabla y+y \partial_{t} A-\beta \partial_{t} \widetilde{u}, \text { in }(0, \infty) \times \Omega, \\
z(t, x)=0 \text { on }(0, \infty) \times \partial \Omega \\
z(0, x)=\left(D \Delta y+\frac{2 D}{\widetilde{u}} \nabla \widetilde{u} \cdot \nabla y+A y(0, .)+\alpha-\beta \widetilde{u}(0, .) \text { on } \Omega .\right.
\end{array}\right.
$$

Using Lemma 2.1, we set $\tilde{z}=\frac{z}{\partial_{t} \widetilde{u}}$ and $w=\partial_{t} \tilde{z}$. We obtain $w(t, x)=0$ on $(0, \infty) \times \partial \Omega$ and:

$$
\begin{aligned}
\partial_{t} w= & D \Delta w+C \cdot \nabla w+E w+\partial_{t}(C) \cdot \nabla \tilde{z}+\partial_{t}(E) \tilde{z} \\
& +2 D \partial_{t}\left(\frac{\nabla \tilde{u}}{\tilde{u}}\right) \cdot \frac{\nabla z}{\partial_{t} \tilde{u}}+\frac{\partial_{t} A}{\partial_{t} \tilde{u}} z+\partial_{t}\left(2 D \partial_{t}\left(\frac{\nabla \tilde{u}}{\tilde{u}}\right) \frac{1}{\partial_{t} \tilde{u}}\right) \cdot \nabla y+\partial_{t}\left(\frac{\partial_{t} A}{\partial_{t} \tilde{u}}\right) y,
\end{aligned}
$$

where $C$ and $E$ are functionals depending on $\tilde{u}$ and its time and space derivatives until order two.

Given any couple $0<\tau_{0}<\tau_{1}$ and $0<\delta<\tau_{0}$, we set $Q_{\omega_{i}}=\left[\tau_{i}-\delta, \tau_{i}+\delta\right] \times \omega$ and $Q_{i}=\left[\tau_{i}-\delta, \tau_{i}+\delta\right] \times \Omega$. Given a function $\zeta(x)$, defined on $C^{2}(\bar{\Omega})$ such that $\zeta(x)>0$ on $\Omega, \zeta(x)=0$ on $\partial \Omega,|\nabla \zeta|>0$ on $\overline{\Omega \backslash \omega}$ and some constant $K>0$, we may also define:

$$
\varphi_{i}(x, t)=\frac{e^{\lambda \zeta(x)}}{\left(t-\left(\tau_{i}-\delta\right)\right)\left(\tau_{i}+\delta-t\right)}, \quad \eta_{i}(x, t)=\frac{e^{2 \lambda K}-e^{\lambda \zeta(x)}}{\left(t-\left(\tau_{i}-\delta\right)\right)\left(\tau_{i}+\delta-t\right)}
$$

for $i=0,1$. Note that $\eta_{0}\left(\tau_{0},.\right)=\eta_{1}\left(\tau_{1},.\right)$ on $\Omega$. 
Let us recall a classical Carleman estimate (see [5]):

Theorem 2.3 Let $\rho \in \mathbb{R}, 0<\tau_{0}<\tau_{1}$ and $0<\delta<\tau_{0}$. Then it exists a constant $K>0$, a function $K<\zeta(x)<2 K$ in $C^{2}(\bar{\Omega}), \lambda_{0} \geq 0, s_{0}>0$ and a positive constant $C_{0}$ such that, for any $\lambda \geq \lambda_{0}$, any $s \geq s_{0}$, and any function $q \in C^{2}\left(\overline{Q_{i}}\right)$ with $q \equiv 0$ on $\left[\tau_{i}-\delta, \tau_{i}+\delta\right] \times \partial \Omega$, the following estimate holds:

$$
I_{i}(\rho, q) \leq C_{0} s\left[\int_{Q_{\omega_{i}}} e^{-2 s \eta_{i}} \lambda^{4}\left(s \varphi_{i}\right)^{\rho+3}|q|^{2} d t d x+\int_{Q_{i}} e^{-2 s \eta_{i}}\left(s \varphi_{i}\right)^{\rho}\left|\partial_{t} q-\Delta q\right|^{2} d t d x\right],
$$

where

$$
\begin{aligned}
I_{i}(\rho, q)= & s \int_{Q_{i}} e^{-2 s \eta_{i}}\left(s \varphi_{i}\right)^{\rho-1}\left(\left|\partial_{t} q\right|^{2}+|\Delta q|^{2}\right) d t d x \\
& +s^{2} \lambda^{2} \int_{Q_{i}} e^{-2 s \eta_{i}}\left(s \varphi_{i}\right)^{\rho+1}|\nabla q|^{2} d t d x+s \lambda^{4} \int_{Q_{i}} e^{-2 s \eta_{i}}\left(s \varphi_{i}\right)^{\rho+3}|q|^{2} d t d x .
\end{aligned}
$$

Using (7) applied to the solution $y$ of (4), together with Lemmata 2.1 and 2.2 we get that, for any $0<\tau_{0}<\tau_{1}, 0<\delta<\tau_{0}, \lambda_{0} \leq \lambda$, and for $s$ large enough, it exist $C(s, \lambda)>0$ and $C>0$ such that, independently of the choice of $(\tilde{\mu}, \tilde{\gamma}) \in M \times \Gamma$,

$$
I_{i}(0, y) \leq C(s, \lambda) \int_{Q_{\omega_{i}}} \varphi_{i}^{3}|y|^{2} e^{-2 s \eta_{i}} d t d x+C s \int_{Q_{i}}|\alpha-\beta \tilde{u}|^{2} e^{-2 s \eta_{i}} d t d x
$$

Similarly, using (7) applied to the solution $z$ of (5) and Lemma 2.1 of [8], we get that it exist $C(s, \lambda)>0$ and $C>0$ such that:

$$
I_{i}(0, z) \leq C(s, \lambda) \int_{Q_{\omega_{i}}} \varphi_{i}^{3}|z|^{2} e^{-2 s \eta_{i}} d t d x+C s \int_{Q_{i}}|\beta|^{2} e^{-2 s \eta_{i}} d t d x+C(s, \lambda) \|\left(y\left(\tau_{i}, .\right) \|_{H^{1}(\Omega)}^{2} .\right.
$$

Then, from (6) and using once more Lemma 2.1 of [8] we can write:

$$
I_{i}(0, w) \leq C(s, \lambda) \int_{Q_{\omega_{i}}} e^{-2 s \eta_{i}} \varphi_{i}^{3}\left(|w|^{2}+|z|^{2}\right) d t d x+C \frac{1}{\lambda^{2}} \int_{Q_{i}}|\beta|^{2} e^{-2 s \eta_{i}} d t d x+C(s, \lambda) \|\left(y\left(\tau_{i}, .\right) \|_{H^{1}(\Omega)}^{2} .\right.
$$

Multiplying (4) by $e^{-s \eta_{0}}$, evaluating at time $t=\tau_{0}$ and taking the $L^{2}$ norm in space we can get an upper bound for $\left\|\alpha e^{-s \eta_{0}\left(\tau_{0}, .\right)}\right\|_{L^{2}(\Omega)}^{2}$. Then, using Carleman estimates (8), (9) and (10) we also obtain an upper bound for $\left\|\left(\alpha-\beta \tilde{u}\left(\tau_{1}, .\right)\right) e^{-s \eta_{1}\left(\tau_{1}, .\right)}\right\|_{L^{2}(\Omega)}^{2}$. Combining these two upper bounds, and using the fact that $\eta_{0}\left(\tau_{0},.\right)=\eta_{1}\left(\tau_{1},.\right)$, we obtain, for sufficiently large $s$, the existence of $C>0$, such that:

$$
\begin{aligned}
& \left\|\alpha e^{-s \eta_{0}\left(\tau_{0}, .\right)}\right\|_{L^{2}(\Omega)}^{2}+\left\|\beta \tilde{u}\left(\tau_{1}, .\right) e^{-s \eta_{0}\left(\tau_{0}, .\right)}\right\|_{L^{2}(\Omega)}^{2} \leq \\
& C \int_{Q_{\omega_{1}}} \varphi_{1}^{3} e^{-2 s \eta_{1}}|z|^{2} d t d x+C \int_{Q_{\omega_{0}}} \varphi_{0}^{3} e^{-2 s \eta_{0}}\left(|w|^{2}+|z|^{2}\right) d t d x \\
& +C\left\|y\left(\tau_{0}, .\right)\right\|_{H^{2}(\Omega)}^{2}+C\left\|y\left(\tau_{1}, .\right)\right\|_{H^{2}(\Omega)}^{2}+6 \sup _{\bar{\Omega}}\left|\tilde{u}\left(\tau_{0}, .\right)\right|^{2}\left\|\beta e^{-s \eta_{0}\left(\tau_{0}, .\right)}\right\|_{L^{2}(\Omega)}^{2} .
\end{aligned}
$$

We then use Lemma 1.1 to find two times $t_{0}$ and $t_{1}$ such that $6 \sup _{x \in \bar{\Omega}} \tilde{u}\left(t_{0}, x\right)<\inf _{x \in \bar{\Omega}} \tilde{u}\left(t_{1}, x\right)$, and we fix $\delta \in\left(0, t_{0}\right)$. We finally obtain the existence of a constant $C>0$ such that, for any couple $(\tilde{\mu}, \tilde{\gamma}) \in M \times \Gamma$,

$$
\begin{aligned}
\|\alpha\|_{L^{2}(\Omega)}^{2}+\|\beta\|_{L^{2}(\Omega)}^{2} & \leq C \int_{t_{0}-\delta}^{t_{1}+\delta} \int_{\omega} \varphi_{\max }^{3} e^{-2 s \eta_{\min }}\left(|z|^{2}+|w|^{2}\right) d t d x+C\left\|y\left(t_{0}, .\right)\right\|_{H^{2}(\Omega)}^{2}+C\left\|y\left(t_{1}, .\right)\right\|_{H^{2}(\Omega)}^{2} \\
& \leq C\left(\|u-\tilde{u}\|_{H^{2}\left(\left(t_{0}-\delta, t_{1}+\delta\right), L^{2}(\omega)\right)}^{2}+\left\|(u-\tilde{u})\left(t_{0}, .\right)\right\|_{H^{2}(\Omega)}^{2}+\left\|(u-\tilde{u})\left(t_{1}, .\right)\right\|_{H^{2}(\Omega)}^{2}\right)
\end{aligned}
$$

where $\eta_{\min }(.,)=.\min _{\left(t_{0}-\delta, t_{1}+\delta\right) \times \omega}\left(\eta_{0}, \eta_{1}\right)(.,$.$) and \varphi_{\max }(.,)=.\max _{\left(t_{0}-\delta, t_{1}+\delta\right) \times \omega}\left(\varphi_{0}, \varphi_{1}\right)(.,$.$) .$ 


\section{References}

[1] M Cristofol and L Roques. Biological invasions: Deriving the regions at risk from partial measurements. Mathematical Biosciences, 215(2):158-166, 2008.

[2] M Cristofol and L Roques. Simultaneous reconstruction of two coefficients in a nonlinear parabolic equation. In preparation, 2010.

[3] M Choulli and M Yamamoto. Some stability estimates in determining sources and coefficients. Journal of Inverse and Ill-Posed Problems, 14:355-373, 2006.

[4] M El Smaily, F Hamel, and L Roques. Homogenization and influence of fragmentation in a biological invasion model. Discrete and Continuous Dynamical Systems, Series A, 25:321-342, 2009.

[5] E Fernández-Cara and S Guerrero. Global Carleman inequalities for parabolic systems and application to controllability. SIAM Journal on Control and Optimization, 45:1395-1446, 2006.

[6] R A Fisher. The wave of advance of advantageous genes. Annals of Eugenics, 7:335-369, 1937.

[7] A Friedman. Partial differential equations of parabolic type. Prentice-Hall, Englewood Cliffs, NJ, 1964.

[8] M V Klibanov. Global uniqueness of a multidimensional inverse problem for a nonlinear parabolic equation by a Carleman estimate. Inverse Problems, 20:1003-1032, 2004.

[9] A N Kolmogorov, I G Petrovsky, and N S Piskunov. Étude de l'équation de la diffusion avec croissance de la quantité de matière et son application à un problème biologique. Bulletin de l'Université d'État de Moscou, Série Internationale A, 1:1-26, 1937.

[10] L Roques, M-A Auger-Rozenberg, and A Roques. Modelling the impact of an invasive insect via reaction-diffusion. Mathematical Biosciences, 216(1):47-55, 2008.

[11] L Roques and M D Chekroun. On population resilience to external perturbations. SIAM Journal on Applied Mathematics, 68(1):133-153, 2007.

[12] L Roques and M Cristofol. On the determination of the nonlinearity from localized measurements in a reaction-diffusion equation. Nonlinearity, 23:675-686, 2010.

[13] L Roques and F Hamel. Mathematical analysis of the optimal habitat configurations for species persistence. Mathematical Biosciences, 210(1):34-59, 2007.

[14] L Roques, A Roques, H Berestycki, and A Kretzschmar. A population facing climate change: joint influences of Allee effects and environmental boundary geometry. Population Ecology, 50(2):215-225, 2008.

[15] L Roques and R S Stoica. Species persistence decreases with habitat fragmentation: an analysis in periodic stochastic environments. Journal of Mathematical Biology, 55(2):189-205, 2007.

[16] J G Skellam. Random dispersal in theoretical populations. Biometrika, 38:196-218, 1951.

[17] N Shigesada, K Kawasaki, and E Teramoto. Traveling periodic-waves in heterogeneous environments. Theoretical Population Biology, 30(1):143-160, 1986.

[18] M Yamamoto and J Zou. Simultaneous reconstruction of the initial temperature and heat radiative coefficient. Inverse Problems, 17:1181-1202, 2001. 